\title{
Benign lesions of mandible-imaging findings using MDCT with histopathological correlation
}

\author{
Ruchi Gupta ${ }^{1 *}$, Sailesh Kumar Mukul ${ }^{2}$, Abhishek Singh ${ }^{2}$, Subhash Kumar ${ }^{1}$, Neetu Sinha ${ }^{1}$ and Ilkan Tatar ${ }^{3}$ \\ ${ }^{1}$ Department of Radiodiagnosis, AIIMS, India \\ ${ }^{2}$ Department of Dentistry, AIIMS, India \\ ${ }^{3}$ Department of Ophthalmology, AIIMS, India
}

\begin{abstract}
Mandibular lesions have a very wide spectrum but overlapping imaging features which pose a great diagnostic challenge. This essay aims to describe the imaging features of the histologically proven benign lesions of the mandible.
\end{abstract}

\section{Introduction}

Mandibular lesions have odontogenic and non-odontogenic origins. Teeth develop both from ectoderm and mesoderm. Enamel develops from ectoderm of the oral cavity and all other tissues from associated mesenchyme [1]. Odontogenic tumors demonstrate interactions between the odontogenic epithelium and odontogenic ectomesenchyme and are typically sub classified by their tissue of origin. Odontogenic tumors usually involve one tooth or specific part of tooth while non-odontogenic lesions have no specific relationship to dentition, hence can involve the bone around two or more teeth.

WHO histological classification of benign odontogenic tumors

Odontogenic epithelium with mature, fibrous stroma without odontogenic ectomesenchyme

1. Ameloblastoma, solid/multicystic type

2. Ameloblastoma, extraosseous/peripheral type

3. Ameloblastoma, desmoplastic type

4. Ameloblastoma, unicystic type

5. Squamous odontogenic tumor

6. Calcifying epithelial odontogenic tumors

7. Adenomatoid odontogenic tumor

8. Keratocystic odontogenic tumor

Odontogenic epithelium with odontogenic ectomesenchyme, with or without hard tissue formation

1. Ameloblastic fibroma

2. Ameloblastic fibrodentinoma

3. Ameloblastic fibro-odontoma

4. Odontoma, complex type

5. Odontoma, compound type

6. Odontoameloblastoma
7. Calcifying cystic odontogenic tumor

8. Dentinogenic ghost cell tumor

Mesenchyme and/or odontogenic ectomesenchyme with or without odontogenic epithelium

1. Odontogenic fibroma

2. Odontogenic myxoma/myxofibroma

3. Cementoblastoma

Bone-related lesions

1. Ossifying fibroma

2. Fibrous dysplasia

3. Osseous dysplasia's

4. Central giant cell lesion (granuloma)

5. Cherubism

6. Aneurysmal bone cyst

7. Simple bone cyst

\section{Subjects and methods}

All suspected patients of Mandibular lesions underwent Computed tomography (CT) FACE on 256 Slice Dual Source SEIMENS CT Scanner. All radiologically diagnosed focal or diffuse suspicious lesions underwent biopsy. All biopsy proven benign Mandibular lesions were included in this article.

${ }^{*}$ Correspondence to: Ruchi Gupta, Department of Radiodiagnosis, AIIMS, Patna, India, Tel: 9718407808, E-mail: drruchigupta28@gmail.com

Key words: benign, mandible, computed tomography, lesions, masses, jaw

Received: August 03, 2018; Accepted: August 20, 2018; Published: August 24, 2018 


\section{Discussion}

\section{Cystic lesions of jaw} [2].

Majority of cysts are odontogenic in origin and are epithelium lined

\section{Radicular cyst (periapical cyst)}

They are the most common odontogenic cyst resulting due to inflammation secondary to caries. The cyst arises from epithelial remnants called rest of Malassez that are located in the periodontal ligament [1]. Infection spreads to the root of tooth resulting in periapical periodontitis, granulomas, abscess and finally a cyst formation. Peak incidence is between 30 and 50 years of age [2]. On radiography, occur as well circumscribed radiolucent lesions at the apex of tooth, usually measuring less than $1 \mathrm{~cm}$, may lead to root resorption in long standing lesions (Figure 1). The term residual cyst should be used when the lesion persists even after tooth extraction.

\section{Dentigerous (follicular) cyst}

It is the second most common odontogenic cyst that develops around the crown of an unerupted tooth, usually the third molar tooth. The enamel epithelium lining the crown of tooth proliferates and secretes fluid. Fluid collects between the layers of epithelium or between epithelium and enamel. The wall of the cyst tends to converge to cement-enamel junction. Hence, presence of cystic cavity with crown inside the cavity is virtually diagnostic $[1,2]$. Displacement of the teeth is seen and resorption is not a feature of follicular cyst. Ameloblastoma, mucoepidermoid tumor and carcinomas may develop in the wall of the cyst [1] (Figures 2 and 3 ).

\section{Lateral periodontal cyst}

It is a developmental cyst that arises from epithelial remnants in periodontal ligament along lateral aspect of the tooth root.

\section{Lateral radicular cyst}

It is analogous to Periapical cyst but occur along lateral aspect of the root.

\section{Non-odontogenic cysts}

Simple/Hemorrhagic/Traumatic bone cyst is a pseudocyst without an epithelial lining that usually is seen in young people in second

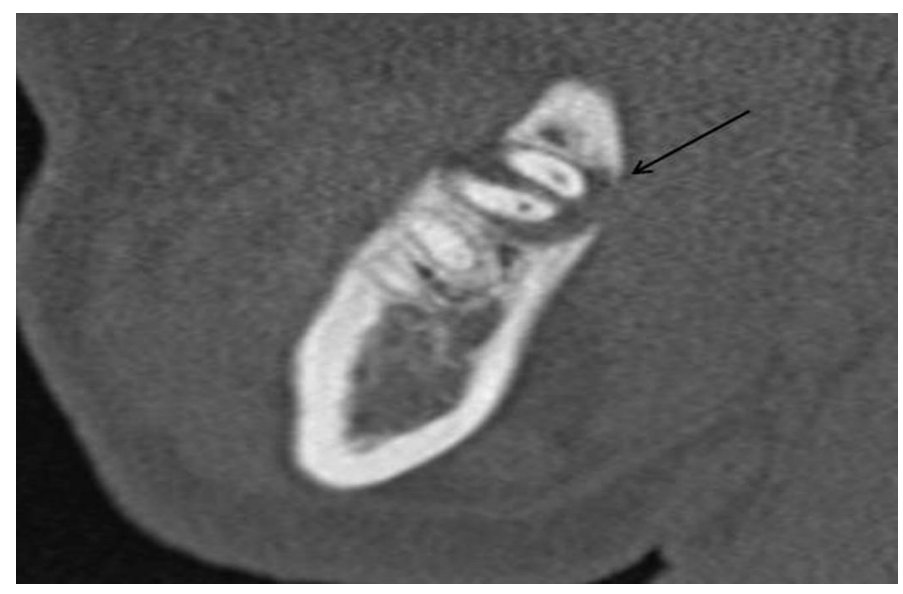

Figure 1. Radicular cyst- Small well-defined cystic lesion at the apex of last molar tooth Cortical thinning of the adjacent mandibular cortex is seen. No evidence of resorption of tooth seen

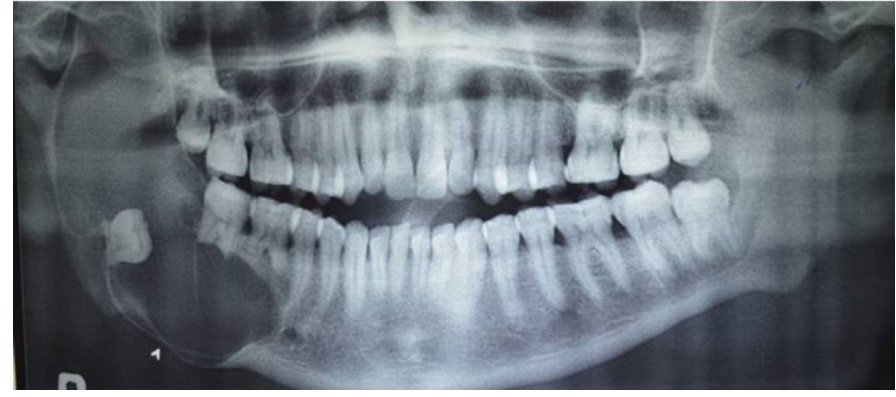

Figure 2. Dentigerous cyst: 24 years old male. Orthopantomogram shows lobulated expansile cystic lesion in body and ramus of mandible on right side extending into the coronoid process. The lesion is associated with impacted 3rd molar and crown of the tooth lies within the cystic cavity. There is marked thinning and remodelling of the adjacent cortex of mandible

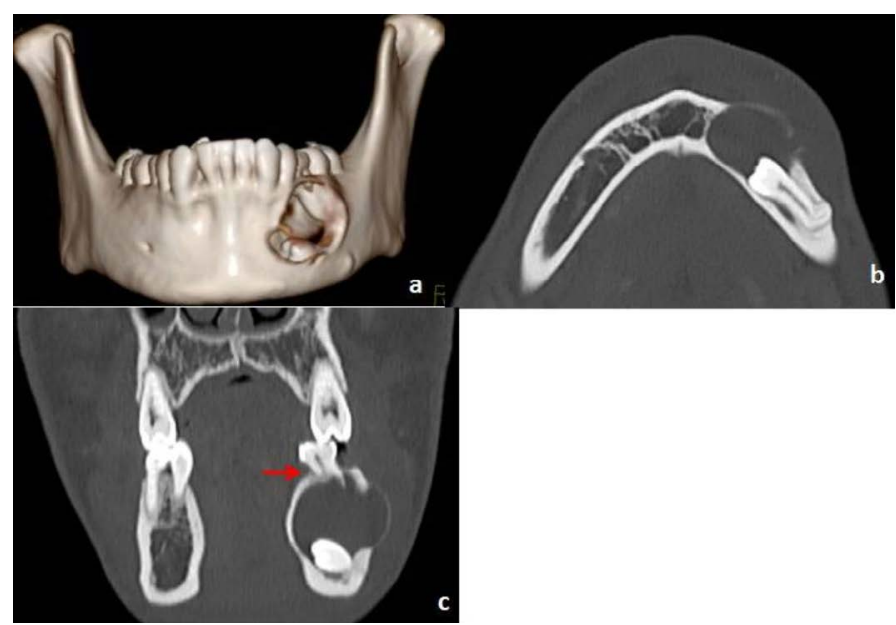

Figure 3. Dentigerous cyst with ectopic tooth: 26-year-old male (a) VRT image shows cystic cavity with cortical defect in body of mandible on left side associated with ectopic tooth in the floor of the cyst. (b) Axial bone window image shows cystic lesion is associated with the crown of the ectopic tooth and root is directed posteriorly. (c) Coronal bone window image shows resorption of the root of adjacent premolar tooth (red arrow)

decade of life. These are usually asymptomatic, discovered incidentally and most commonly located in premolar region rather in incisor area of mandible [1]. On imaging, they are slightly irregular in shape, poorly defined borders; give scalloped appearance between the roots of the teeth. On CT, high density may confirm blood products, but density is variable.

Aneurysmal bone cyst (ABC) is often expansile, multilocular lesion show cortical thinning or destruction and contains blood filled spaces separated by fibrous septa. The appearance is similar as in long bones. On CT/MRI, Presence of fluid levels may be present, strongly suggestive of ABC. In our case, Fluid levels were not seen, however expansile fluid density lesion causing marked bone remodeling and thinning was seen (Figure 4).

Stafne cyst (static bone cavity) is a radiolucency usually located on the medial surface of the posterior mandible near the angle of mandible below the mylohyoid line and mandibular canal. These are more common in males with age range 20-70years. The bone defect usually contains submandibular salivary gland or fatty tissue (Figure 5).

\section{Odontogenic tumors}

Ameloblastoma is a benign odontogenic tumor that arises from ameloblasts (enamel forming cells), has peak incidence in $3^{\text {rd }}$ to $4^{\text {th }}$ 


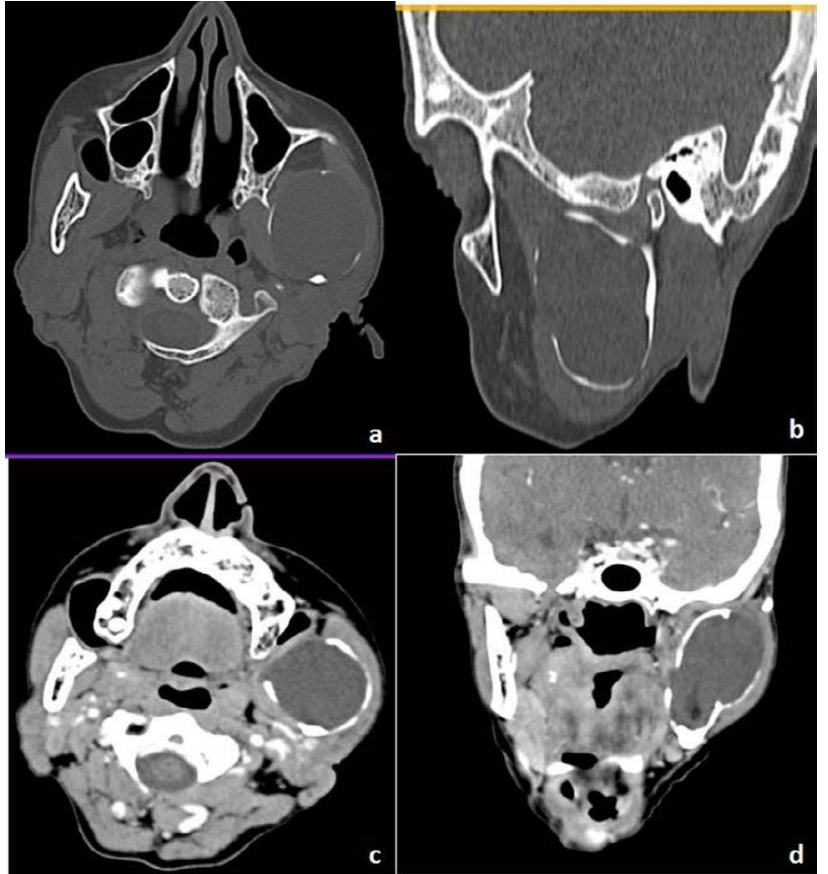

Figure 4. Aneurysmal bone cyst: 26-year-old male (a) Axial (b) Coronal bone window images show well circumscribed expansile cystic lesion in left ramus of mandible extending into the coronoid process and sigmoid notch. Surrounding bone is thinned, remodelled and eroded, however capsule of the lesion appear intact. Post contrast (c) Axial (d) Coronal images show no enhancement on the post contrast study

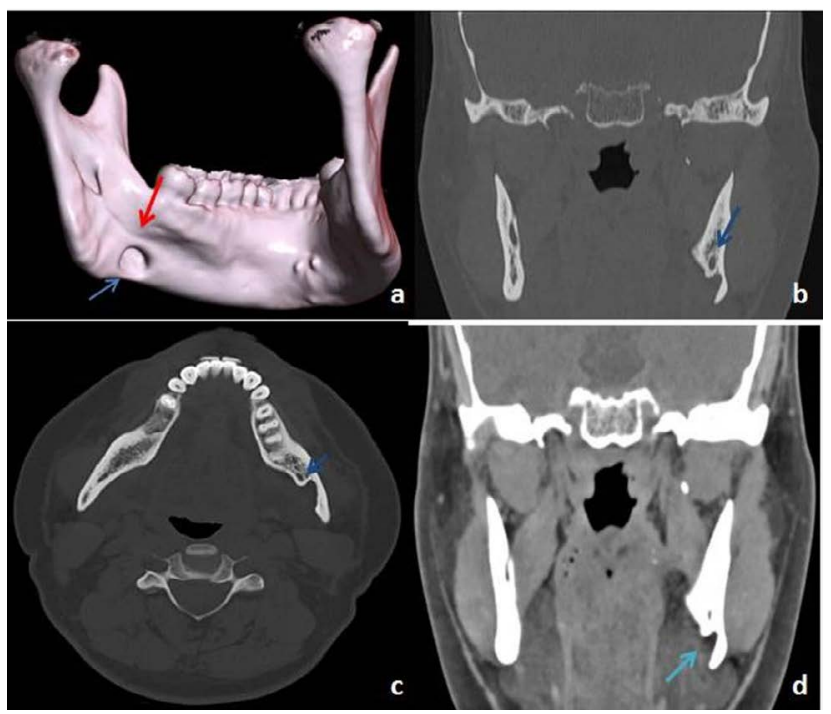

Figure 5. Stafne cyst: 39-year-old male presented with clicking sound in right TMJ, the cyst was an incidental finding. (a) VRT image shows the smooth cortical defect in mandible at the level of angle of mandible (blue arrow) below the myelohyoid line (red arrow). (b, c) Coronal and Axial bone window image shows the cortical defect is in the postero-medial cortex with inferior alveolar nerve superior and anterior to the lesion (blue arrow). (d) Coronal soft tissue image shows a portion of the submandibular gland is within the cystic lumen (light blue arrow)

incidence of life with majority of lesions occur in mandible and in posterior region.

Multiloculated/solid type give honeycombing like appearance, may invade cortex to extend into adjacent tissues, may lead to scalloping and bony expansion. On MRI, mixed solid and cystic components, thick walls, papillary projections with enhancing solid component and septae may be seen. Loss of lamina dura, erosion of tooth apex and displacement of teeth may also be seen. Presence of enhancing soft tissue component and papillary projections differentiates it from other cystic lesions [1] (Figure 6).

The Extra osseous/Peripheral type leads to superficial erosion of the bone due to pressure resorption with rare significant involvement of bone.

Desmoplastic type occurs mainly in anterior mandibular region, has mixed lucent-opaque appearance with ill-defined borders, hence difficult to distinguish from bone related lesions. Unicystic type usually present at a younger age in second decade of life with predilection for posterior mandible [3]. Up to $80 \%$ are associated with an unerupted tooth usually the mandibular third molar. Radiographically, present as well corticated unilocular lesion with other features similar to other classical ameloblastoma (Figures 7 and 8).

Calcifying epithelial odontogenic tumor (Pindborg tumor) is a locally invasive epithelial odontogenic neoplasm made of epithelial cells in fibrous stroma containing acidophilic homogenous structures that often calcify [1]. They most commonly occur between 20 and 60 years of age (mean 40 years) [4]. Many of the lesions are seen in molar and premolar region and half of the cases are associated with crown of an impacted or unerupted tooth [4]. Radiographically, mixed lucent-

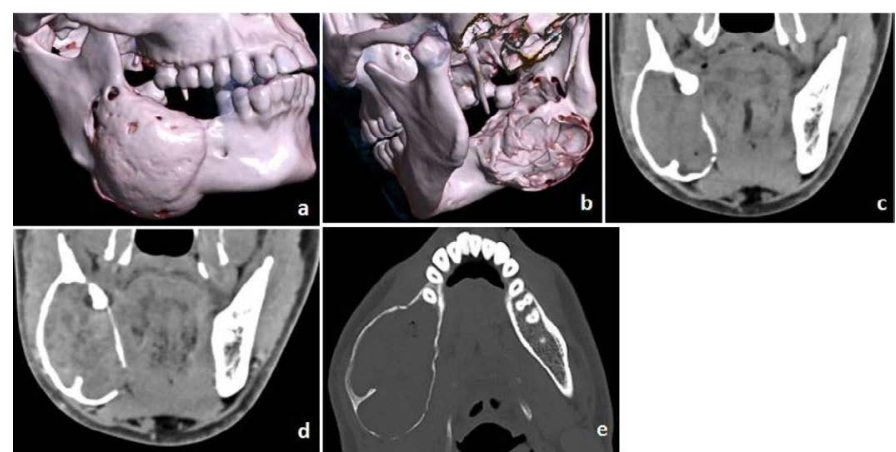

Figure 6. Ameloblastoma: 30-year-old male (a, b) VRT images showing markedly expansile lytic lesion in the body, angle and adjacent ramus of the mandible with multiple site perforated cortices more on lingual than the buccal side. (c) Coronal plain soft tissue (d) Post contrast image shows heterogeneous enhancement of the matrix. Peripheral septa are also seen. (e) Axial bone window image shows thinning and expansion of cortex with septa within

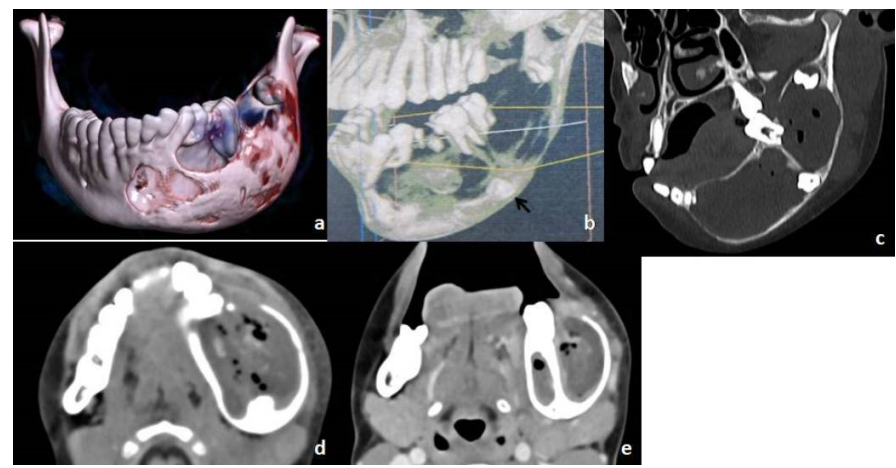

Figure 7. Unicystic ameloblastoma: 10-year-old female, swelling in left side of the face for 8 months with intraoral pus discharge (a, b) VRT images (c) Oblique sagittal bone window image showing expansile lytic lesion in the left body, angle and ramus mandible with perforated cortex at many sites. The impacted tooth was seen at the floor of the lesion (black arrow). (d) Axial plain soft tissue (e) Post contrast image shows mild peripheral enhancement, mild hyperdense contents in the matrix and air foci s/o fistulous connection with oral cavity and superimposed infection 


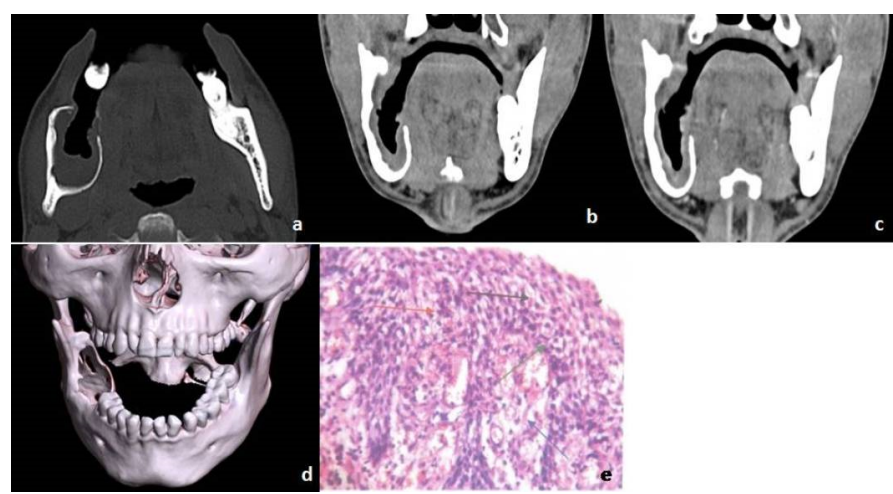

Figure 8. Inflamed Unicystic Ameloblastoma: 36-year-old male (a) Axial bone window, (b) Coronal Soft tissue - An expansile intramedullary lytic lesion seen involving the body, angle, and ramus of mandible eroding the superior border of the mandible and inner cortex. (c) Coronal post-contrast image- The peripheral soft tissue shows mild enhancement. (d) VRT image shows mental foramen is intact. (e) H\&E stained section shows non-keratinized epithelial lining (red arrow), basal amelobastoid (green arrow), inflamed stroma (blue arrow). The differential was Infected KOT

opaque lesion, may be uni or multilocular and has poorly defined margins. Calcified components are seen close to crown of an impacted tooth (Figure 9).

Odontoma are the most common benign odontogenic tumors of epithelial and mesenchymal origin considered as hamartomas. They are developmental lesions as dentin and enamel are laid down in an abnormal pattern. They are made up of various components of teeth (enamel, dentin, cementum or pulp) [5]. They are of two types: Complex and compound. Complex odontomas consist of radio-opaque masses usually associated with an unerupted tooth, most commonly seen in molar and premolar region in mandible, also seen in maxilla. Occasionally, surrounding lucent rim is seen. Compound odontomas contain variable amount of tooth like elements, most commonly seen in incisor- canine region of maxilla (Figure 10).

Adenomatoid odontogenic tumor is an uncommon odontogenic neoplasm more common in young females in second decade of life and maxilla is more commonly involved than mandible often associated with unerupted permanent tooth mimicking dentigerous cyst [6]. The lesions have well demarcated radiolucent centers with punctuate calcifications. If attached to tooth, lesions are found more apically on root, than dentigerous cyst [2] (Figure 11).

Ameloblastic fibroma is a rare odontogenic tumor that resembles the dental papillae and small islands of odontogenic epithelium that resembles the dental lamina. More commonly seen in children with mandible more often affected than maxilla. Radiographically, lesions appear as well-defined to poorly-defined unilocular or multilocular radiolucency [7].

Keratocyst odontogenic tumor is a benign odontogenic tumor with a characteristic parakeratinized stratified squamous epithelium. The lesion is more commonly seen in posterior mandible and occurs in $2^{\text {nd }}$ and $3^{\text {rd }}$ decades of life. The lesions can be multiple in Basal cell nevus syndrome. The lesion can be unilocular or multilocular with smooth scalloped margins, bulging of bony cortex, occur commonly in body and ramus of mandible and may be associated with impacted tooth. The lesion grows along the length of mandible and does not usually resorb teeth roots. On MRI, lesion has cystic appearance with thin wall, heterogeneous intensity of fluid contents and weak contrast enhancement of the cyst wall [1] (Figures 12 and 13).

Odontogenic myxoma is an odontogenic tumor of mesenchymal in origin, seen in $2^{\text {nd }}$ and $3^{\text {rd }}$ decade of life. Mandible and maxilla are both equally involved, and body and ramus are most commonly involved in mandible. The lesion contains stellate and spindle shaped cells with an abundant myxoid or mucoid extracellular matrix. The lesion appears multilocular or unilocular, may give honeycombing like appearance mimicking ameloblastoma and other tumors and may cause extensive destruction of bone with marked cortical expansion $[1,8]$.

Cementoblastoma is a rare odontogenic tumor characterised by cementum like mass connected at the tooth root. More commonly seen in males younger than 20 years of age with predilection for mandibular first molar followed by premolar teeth. Radiographically, they appear as radiopaque mass confluent with the tooth root surrounded by a thin radiolucent periphery fused with roots of a vital teeth.(9)

\section{Bone related lesions}

\section{Ossifying fibroma}

Fibro osseous lesions constitute a spectrum of diseases in which normal bone tissue is replaced by fibroblasts and fibrous tissue with

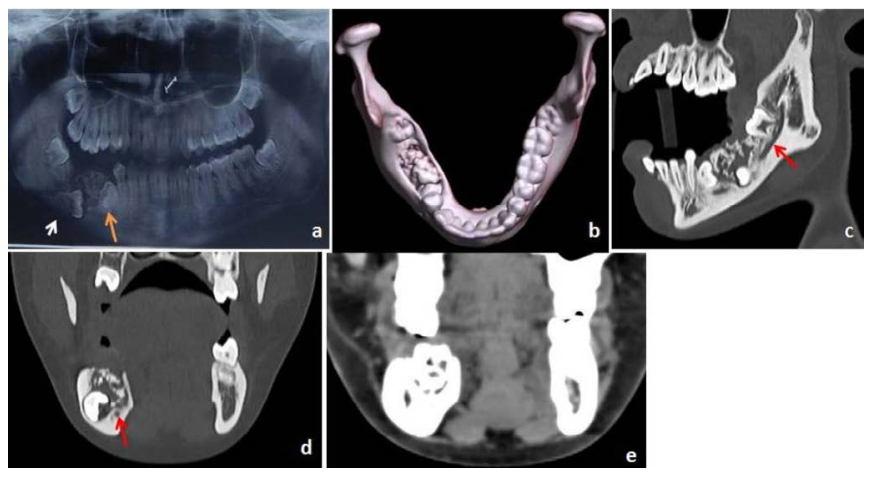

Figure 9. Calcifying epithelial odontogenic tumor (CEOT): 18-year-old female with pain in right lower alveolar region for 1-year (a) Panorex image shows mixed density lesion in right lower alveolar arch, impacted 1st molar tooth oriented horizontally (white arrow) and 2nd premolar displaced inferiorly (orange arrow). (b) VRT image showing the lesion with perforated superior cortex. (c) Oblique sagittal (d) Coronal bone window (e) Soft tissue images show well-defined lesion with inner calcified matrix. Inferior alveolar nerve is just beneath the lesion (red arrow)

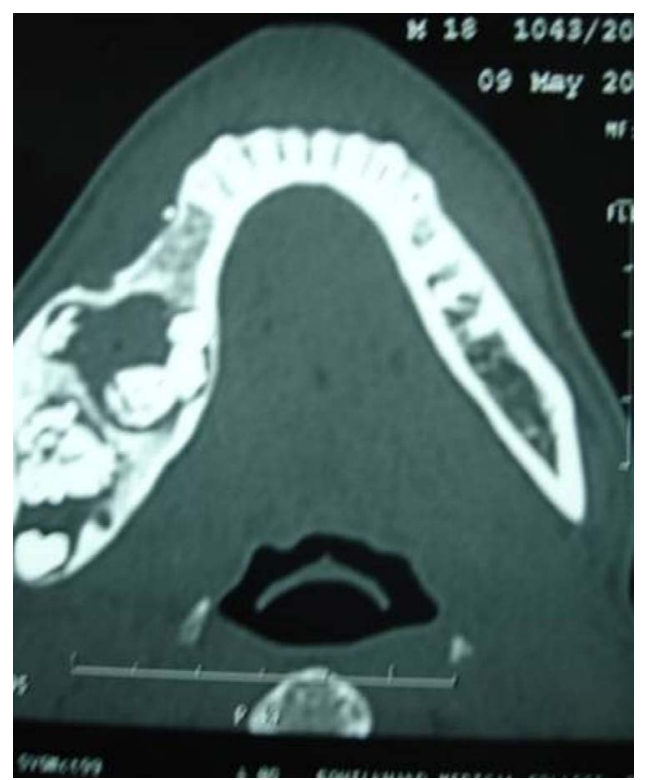

Figure 10. Compound Odontome: 18-year-old male. Well circumscribed radiodense lesions with surrounding cavities, seen in lower alveolar arch posteriorly on the right side with discernible odontogenic elements within 


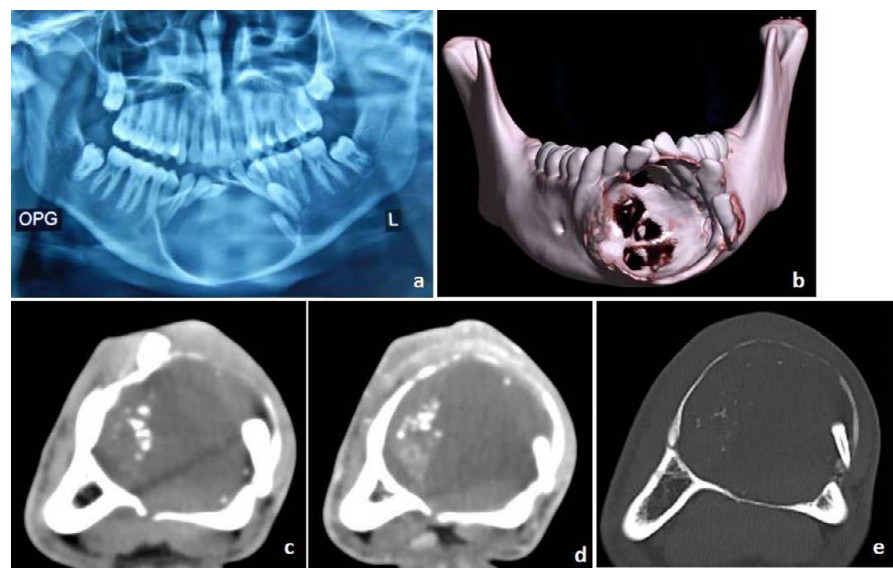

Figure 11. Adenomatoid odontogenic tumor: 18-year-old male with swelling in lower alveolar region for 2 months (a) OPG shows well circumscribed expansile lytic lesion with sclerotic margins in body of mandible in midline with impacted teeth in left lateral wall. (b) VRT image shows both lingual and buccal cortex perforations with displaced and impacted teeth. (c) Axial plain (d) Post contrast image showing specks of calcification within the lesion and mild enhancement of the matrix. (e) Axial bone window image shows remodelled cortex with crown of impacted tooth within the left lateral wall of cyst

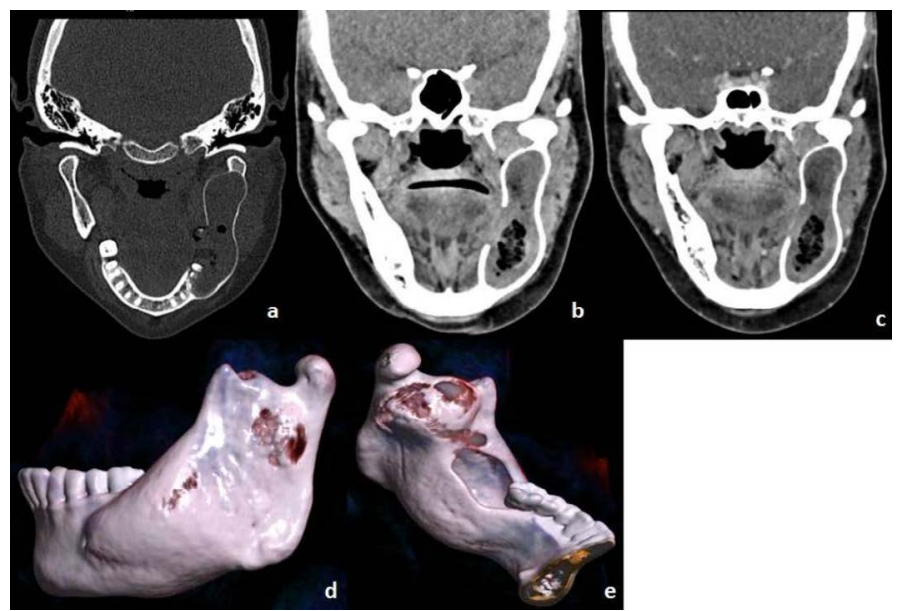

Figure 12. Keratocyst odontogenic tumor: 25-year-old female (a) Coronal Plain bone window and (b) Soft tissue image- An expansile lytic lesion involving left body, ramus of mandible extending up to coronoid process. (c) Post contrast Coronal image- Peripheral subtle enhancement is seen with central fluid contents and air pockets. (d, e) VRT imagesThe lesion is causing cortical thinning with perforations of the buccal and lingual cortex. Medially there is cortical breach with extension of air from the oral cavity into the lesion to suggest fistula formation

presence of mineralized matrix. Fibrous dysplasia, bony dysplasia and ossifying fibroma constitute fibro osseous lesions. First two are reactive lesions while ossifying fibroma is a true neoplasm [10]. They are more common in women in $3^{\text {rd }}$ to $4^{\text {th }}$ decade of life with premolar and molar area more commonly affected [11]. Radiographically, lesion has distinct boundary with normal bone in contrast to fibrous dysplasia. Lesion is lucent in early stages, becomes radiopaque as lesion grows surrounded by a halo of less ossified tissue [1] (Figures 14-16).

\section{Fibrous dysplasia}

Fibrous dysplasia may occur as single or multiple lesions or may be associated with McCune Albright Syndrome. Radiographically, lesion has varied appearance, may show well defined margin with sclerotic rim or ill-defined with non-sclerotic margin, the matrix may vary from mixture of dense to radiolucent areas of fibrosis, homogenously dense, homogenously ground glass or cystic appearance. Most commonly lesion appears as asymmetric homogenous ground glass attenuation that blends with the bone. The disease can also cause bone remodeling, expansion and thing of the cortex [12]. Upward displacement of the inferior alveolar nerve was considered as a unique feature of fibrous dysplasia [13], however in our case postero-inferior displacement of the canal was noted (Figures 17 and 18). MDCT is useful to know the full extent of the lesion and relation with neurovascular structures.

\section{Other benign non-odontogenic tumors}

Osteochondroma (Exostosis) are the bony outgrowth of normal cancellous and compact (Figure 19).

Torus mandibularis is a type of exostosis on lingual surface of mandible.

Osteoma contains is a benign neoplasm of cortical or cancellous bone with most common site is in paranasal sinuses followed by jaw.

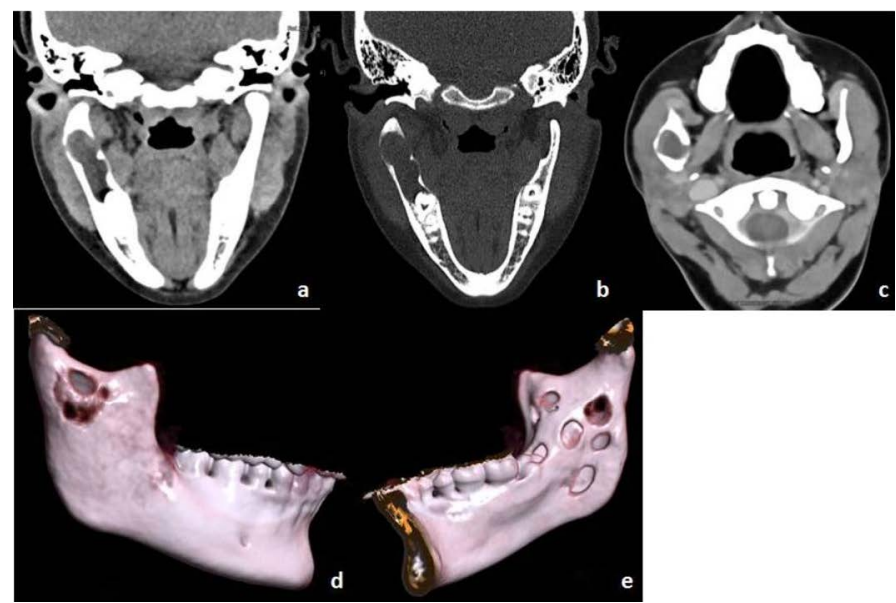

Figure 13. Keratocyst odontogenic tumor (KOT): 22-year-old female (a) Coronal Plain Soft tissue window and (b) Bone window- Hypo dense intramedullary lytic expansile lesion seen involving the right ramus of mandible superiorly reaching up to sigmoid notch and inferiorly up to the 2nd molar tooth. (c) Post contrast axial image- Thin peripheral rim of enhancement is seen. (d, e) Volume rendered images showing breach of cortex both involving lingual and buccal cortex. No periosteal reaction or internal calcification seen

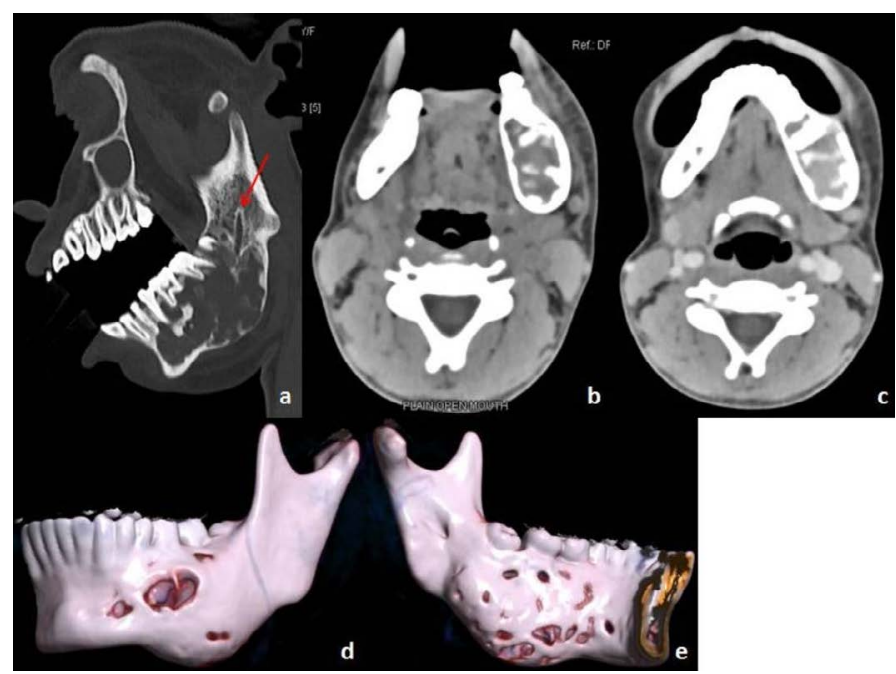

Figure 14. Ossifying Fibroma: 15 -year-old female with swelling over left side of face for 1 year (a) Oblique sagittal bone window, (b) Axial Plain soft tissue image- An expansile lytic lesion involving left half of body and angle of mandible, roots of adjacent molars and premolars lie within the lesion. Inferior alveolar nerve is seen adjacent to the lesion (red arrow). (d, e) VRT images- shows thinning and erosion of both buccal and lingual cortex. (c) Axial post-contrast image- Minimally enhancing hyperdense soft tissue component seen within the lesion 


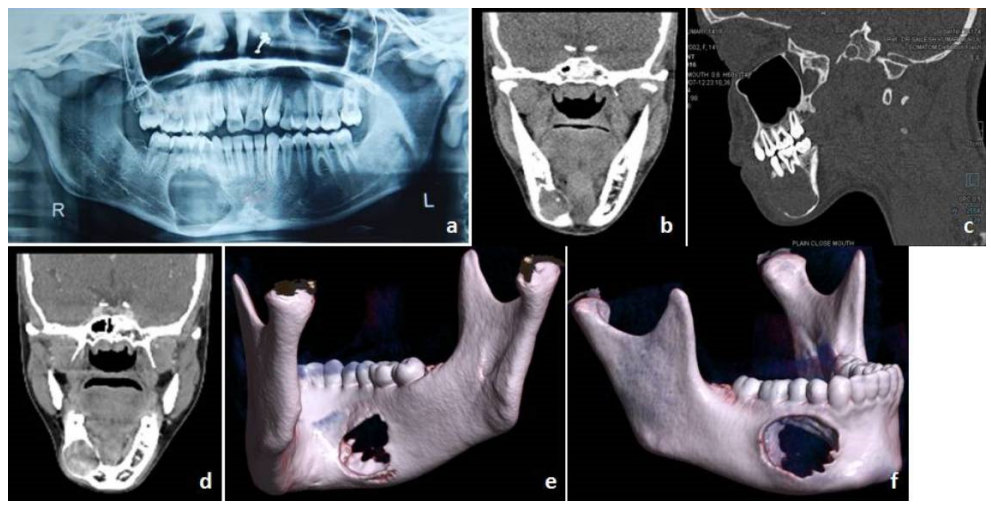

Figure 15. Ossifying fibroma: 14-year-old female (a) Orthopentogram shows well circumscribed lytic lesion in body of mandible on right side causing mildly displaced roots of 1st molar and premolar teeth. (b) Axial Plain CT image shows mildly hyperdense matrix of lesion. (c) Sagittal Bone window CT image shows thinning of surrounding bone and roots of adjacent teeth in peripheral portion of lytic lesion. (d) Post contrast Coronal image shows mild heterogeneous enhancement of the lesion. (e, f) VRT images show smooth cortical defect in both buccal and lingual cortex

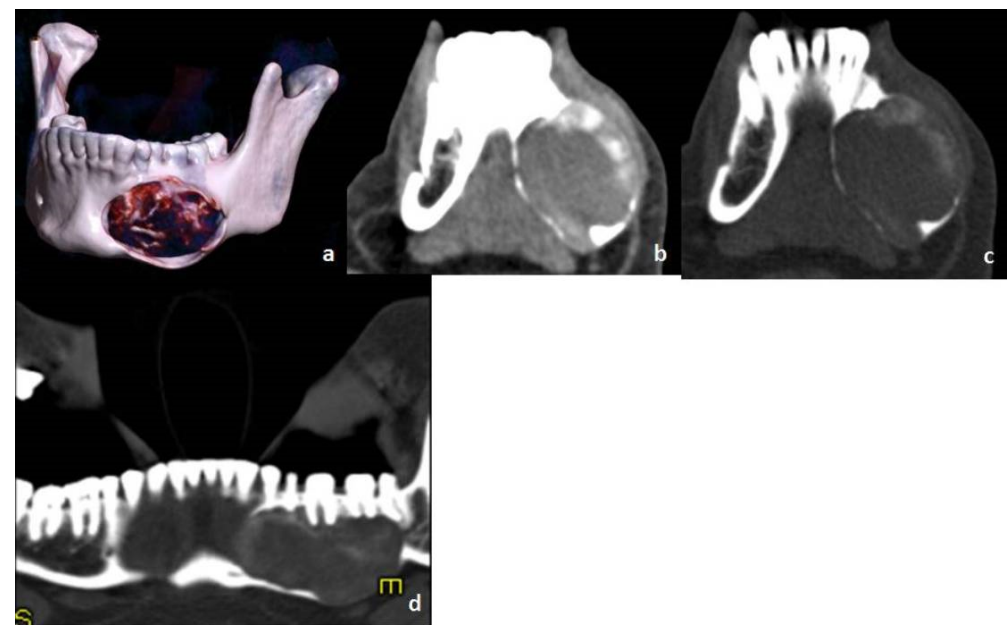

Figure 16. Ossifying fibroma: 36-year-old female with left facial swelling for 1-year (a) VRT image shows well defined expansile lytic lesion in left body region of mandible with adjacent teeth appearing normal. (b) Axial soft tissue (c) Bone window images show lytic expansile lesion with peripheral calcified matrix and thinned out cortex. (d) CT cropped Panorex image shows hyperdense matrix of lesion. Root of adjacent teeth appear mildly displaced but not resorbed

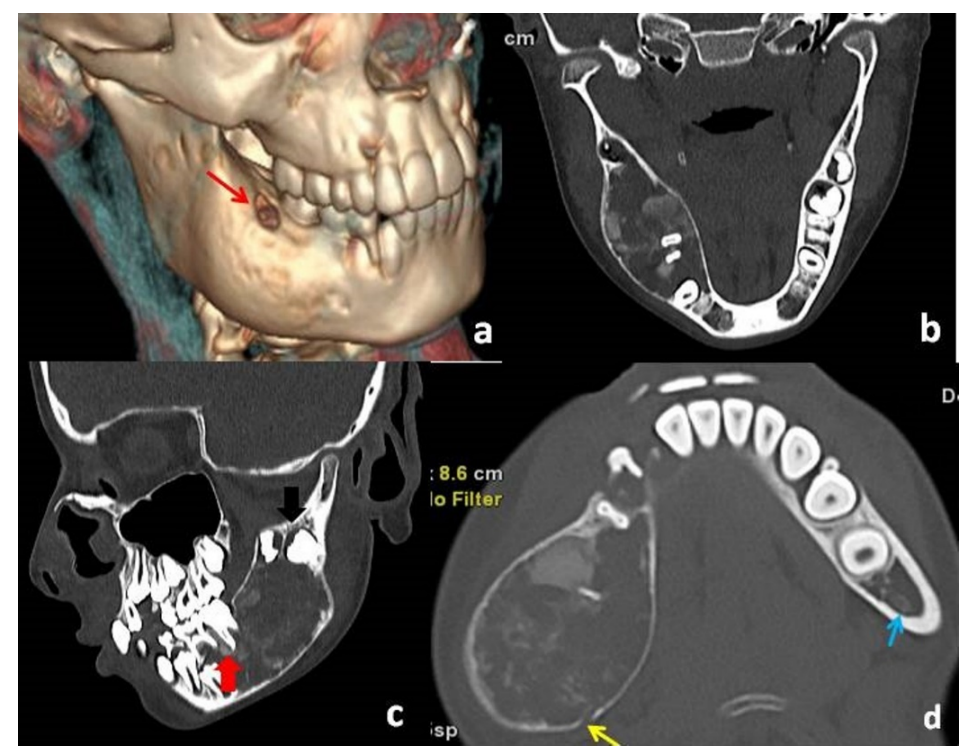

Figure 17. Fibrous dysplasia in a 10-year-old girl. (a) Volume rendered image shows expansile lesion involving posterior body of mandible. Small perforation in buccal cortex seen superiorly (red thin arrow). (b, c) Reformatted Coronal and Sagittal bone window images show mixed lucent and dense matrix giving ground glass appearance with expansile and thinned out cortex. Root of $1^{\text {st }}$ molar and 2 premolars are within the matrix of lesion (red arrow) with displacement of unerupted $2^{\text {nd }}$ and $3^{\text {rd }}$ molar tooth (black arrow). No e/o resorption of root seen. (d) Axial bone window image shows posterior-inferiorly displaced Inferior alveolar nerve on right side (yellow arrow) while normal nerve on left side (blue arrow) 


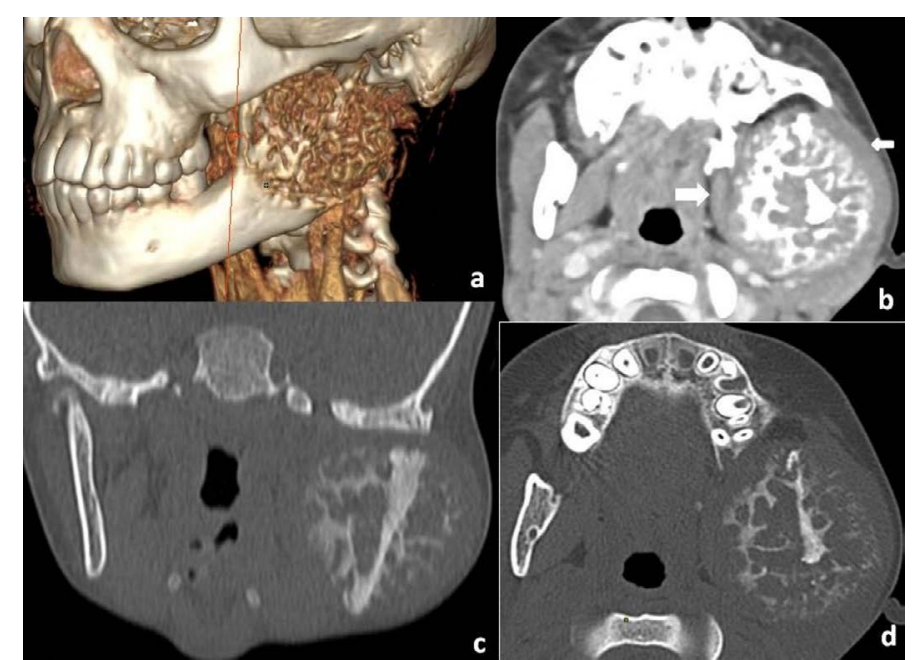

Figure 18. Fibrous dysplasia in a 6-year-old male. (a) Volume rendered image shows expansile lytic lesion with bubbly appearance involving ramus, condyle and coronoid process of mandible. (b) Contrast enhanced Axial CT image shows mild enhancement of the matrix, with sunray appearance of periosteal reaction, however show peripheral somewhat lucent circumscribed margin. The medial pterygoid and masseter muscle are displaced and stretched by the lesion (white arrow). (c, d) Coronal and Axial bone window images show matrix which is in mixed phase, with both lytic and ossified matrix. First differential was fibro osseous lesion followed by osteosarcoma due to mixed features of both entities. It was proven fibrous dysplasia histopathologically

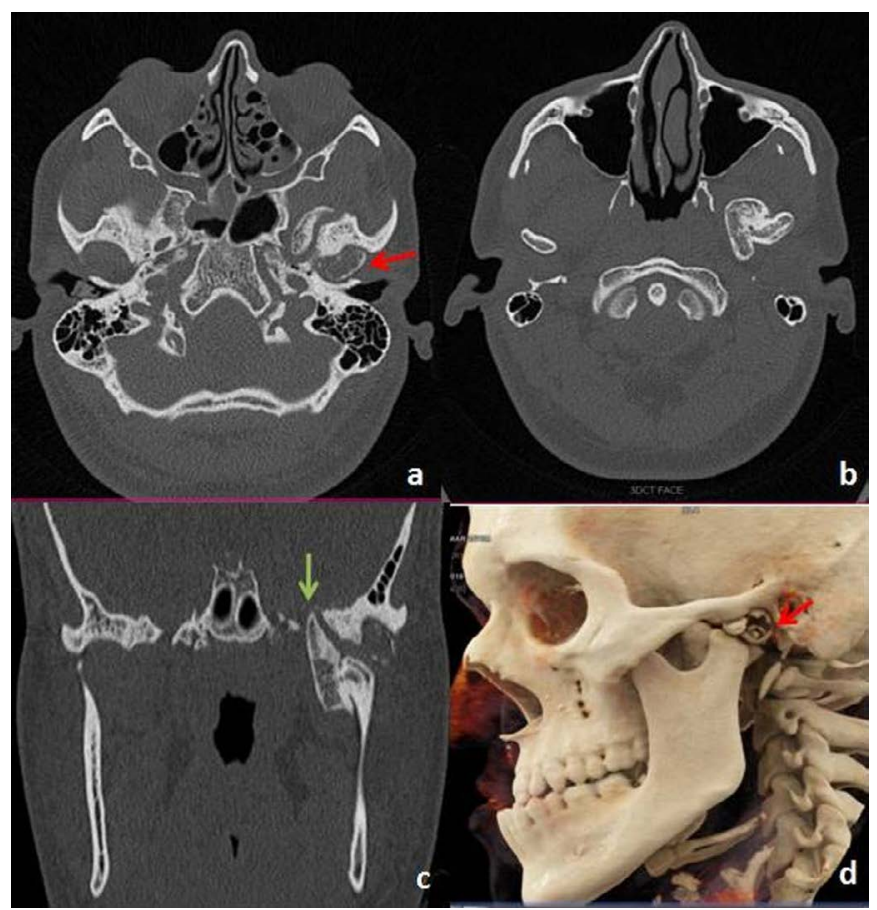

Figure 19. Osteochondroma: 26-year-old male ( $(\mathrm{a}, \mathrm{b})$ Axial bone window images show bony outgrowth from the head of the condyle on the left side with cortex and medulla contiguous with condyle. Partially cystic component of the growth is within the glenoid fossa (red arrow). (c) Coronal bone window image shows bony outgrowth impinging upon middle cranial fossa and foramen ovale (green arrow). (d) VRT image shows osteochondroma from condyle of left mandible with its cystic component in the glenoid fossa

Central giant cell granuloma is most commonly seen in females in $2^{\text {nd }}$ and $3^{\text {rd }}$ decade of life, more common in anterior mandible between $2^{\text {nd }}$ molar and $2^{\text {nd }}$ premolar and tend to cross midline. Radiographically, lesion is often multilocular lucent with honeycombing appearance, thin bony septae but may have unilocular appearance. Lesions often are expansile, can cause tooth displacement and resorption and may produce ground glass opacities and occasional calcification [1].

\section{Paget's disease}

Radiographically, depending upon the stage of progression, there can be punched out radiolucent lesions, mixed areas and more sclerotic areas. In the mandible, both rarefaction and sclerosis are common. In our case, mixed lytic and ground glass opacity is seen with multiple areas of perforated cortex and air foci within to suggest secondary infection [1] (Figure 20).

Hemangioma is a benign neoplasm of proliferating endothelial cells. They are more common in females and rarely involve jaw. Radiographically, appear as radiolucent lesions and may appear multilocular in shape. Doppler may be useful, and angiography shows prolonged parenchymal staining and tissue blush. In our case, hyperdense soft tissue component was seen within the lytic component of the lesion which may suggest locule of blood (Figure 21). 


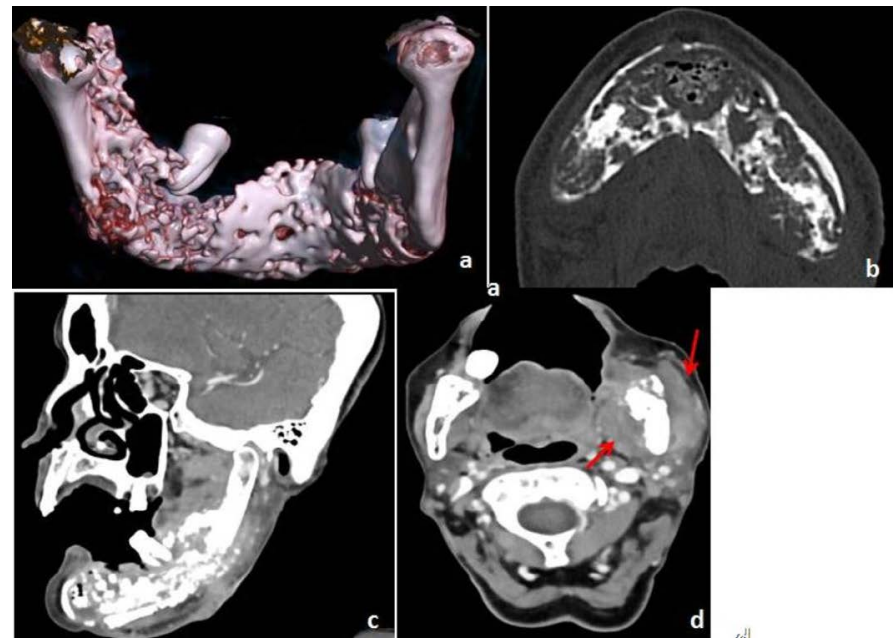

Figure 20. Paget's disease. 80-year-old female (a) VRT (b) Axial bone window image shows multiple intramedullary lytic areas as well as ground glass opacity in body, left ramus and coronoid process of mandible and lower alveolar arch with multiple areas of buccal and lingual cortical breach with presence of air foci within the lesion to suggest secondary infection. (c) Post contrast oblique sagittal image shows mild heterogeneous enhancement of the lesion. (d) Axial post contrast axial image shows bulky left masseter and medial pterygoid muscles to suggest secondary infection (red arrow).
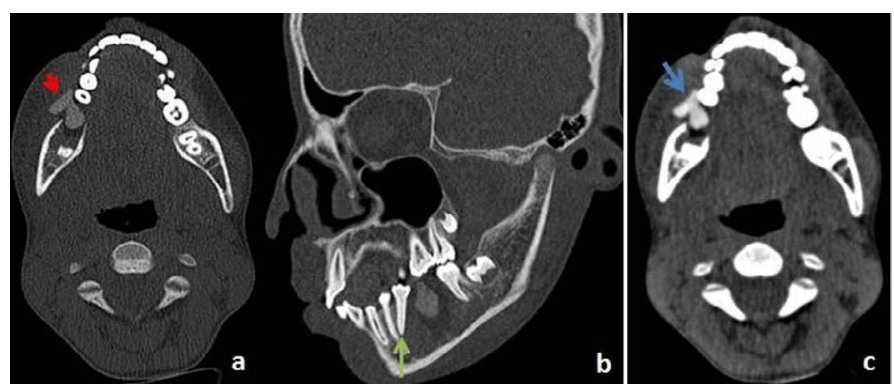

Figure 21. Intraosseous hemangioma: 12-year-old female (a) Axial bone window (b) Oblique sagittal bone window image shows lytic expansile lesion with hyperdense soft tissue within and breach in both lingual and buccal cortex. There is displacement of 2nd premolar and molar tooth (green arrow). The 1st molar tooth is missing. (c) Axial soft tissue image shows lobulated hyperdense component of the lesion projecting out from the buccal cortex (blue arrow)

\section{Neurogenic tumors}

Radiographically, schwannoma arising from inferior alveolar nerve lesions may present as radiolucency which can lead to widening and expansion of inferior alveolar canal. Neurofibroma adjacent to bone may produce pressure defect on the surface of bone.

\section{Inflammatory conditions}

Osteoradionecrosis may occur as a sequel to heavy irradiation which leads to painful necrosis followed by sequestration of bone. Radiographically, in late stages, ill-defined radiolucent areas giving moth eaten appearance with areas of sequestra and variable areas of cortical breach may develop.

Osteomyelitis may develop in jaw secondary to odontogenic infection. The presentation and radiographic findings are similar as in other parts of body.

\section{Conclusion}

History, clinical presentation and imaging play a key role in diagnosis of benign lesions of Mandible. Although CT findings may not always provide confirmatory diagnosis but narrow down the differential diagnosis and provide a definite road map to the surgeon.

\section{References}

1. Som PM, Curtin HD (2011) Cysts, Tumors, and Nontumorous Lesions of the Jaw. (5th edn), Head and neck imaging. St. Louis: Mosby Inc: 1476-1546.

2. Dunfee BL, Sakai O, Pistey R, Gohel A(2006) Radiologic and pathologic characteristics of benign and malignant lesions of the mandible. Radiographics 26: 1751-1768. [Crossref]

3. Hsu MH, Chiang ML, Chen JK (2014) Unicystic ameloblastoma. J Dent Sci 9: 407 411 .

4. Singh N, Sahai S, Singh S, Singh S (2011) Calcifying epithelial odontogenic tumor (Pindborg tumor). Natl J Maxillofac Surg 2: 225-227.

5. Satish V, Prabhadevi MC, Sharma R3 (2011) Odontome: A Brief Overview. Int J Clin Pediatr Dent 4: 177-185. [Crossref]

6. Handschel JG, Depprich RA, Zimmermann AC, Braunstein S, Kübler NR (2005) Adenomatoid odontogenic tumor of the mandible: review of the literature and report of a rare case. Head Face Med 1: 3. [Crossref]

7. Lalitha C, Goyal S, Perka L, Kodangal S (2016) Ameloblastic fibroma. J Indian Acad Oral Med Radiol 28: 199-202.

8. Manne RK, Kumar VS, Venkata Sarath P, Anumula L, Mundlapudi S, et al. (2012) Odontogenic myxoma of the mandible. Case Rep Dent 2012: 214704. [Crossref]

9. Sankari LS, Ramakrishnan K (2011) Benign cementoblastoma. J Oral Maxillofac Pathol 15: 358-360. [Crossref]

10. de Andrade M, Silva-Sousa YT, Marques MF, Pontual ML, Ramos-Perez FM, et al (2013) Ossifying fibroma of the jaws: a clinicopathological case series study. Braz Dent J 24: 662-666. [Crossref]

11. Curé JK, Vattoth S, Shah R (2012) Radiopaque jaw lesions: an approach to the differential diagnosis. Radiographics 32: 1909-1925. [Crossref]

12. Nityasri V, Haris PS, Bose T, Balan A (2011) Fibrous dysplasia-a 13-year retrospective radiographic analysis in a south Indian population. Dentomaxillofac Radiol 40: 282289. [Crossref]

13. Petrikowski CG, Pharoah MJ, Lee L, Grace MG (1995) Radiographic differentiation of osteogenic sarcoma, osteomyelitis, and fibrous dysplasia of the jaws. Oral Surg Oral Med Oral Pathol Oral Radiol Endod 80: 744-750. [Crossref]
Copyright: (C)2018 Gupta R. This is an open-access article distributed under the terms of the Creative Commons Attribution License, which permits unrestricted use, distribution, and reproduction in any medium, provided the original author and source are credited. 